\title{
INVESTIGATION OF SOLUTIONS TO THE FRACTIONAL INTEGRO-DIFFERENTIAL EQUATIONS OF BRATU-TYPE USING LEGENDRE WAVELETS METHOD
}

\author{
M. FELAHAT, N. KADKHODA, AND M. FEČKAN
}

Received 15 June, 2019

\begin{abstract}
In this study, Legendre wavelets has been applied to solve the fractional integrodifferential equations of Bratu-type. In this method, Legendre wavelet operational matrix and numerical integration techniques have been used. Finally, this method is used for solving some examples to illustrate the simplicity of the suggested method.
\end{abstract}

2010 Mathematics Subject Classification: 35R09; 35R11

Keywords: Volterra integral equation, Legendre wavelets, numerical integration methods, Bratutype equations

\section{INTRODUCTION}

Many scientific and engineering problems, including a fractional phenomenon so far are huge, and still are growing. Recently, advances of fractional derivatives and integrals are illustrated by applications in fluid mechanics, viscoelasticity, tracer transfer in underground water, mathematical biology and physics[6, 19]. Recently, fractional behavior of different kinds of dynamical problems has been represented by findings many researchers. This suggests that fractional calculus has an effective role for describing the dynamical problems[4,5]. Several numerical techniques have been introduced for the numerical solutions of fractional differential equations(FDEs), such as Dehghan et al. [8] studied the homotopy analysis method to fractional differential equations, Odibat and Momani [16] used generalized differential transform method to solve the numerical solution of FDEs, Eslahchi et al. [9] applied the collocation method for solving nonlinear fractional integro-differential equations, the explicit and implicit Euler methods were used in the advection-diffusion equation of fractional order by Zhuang et al. [22], and many other researches [11].

Bratu's problem is often appeared in many branches of sciences, such as nanotechnology, the fuel ignition model of the thermal combustion theory, and chemical reaction theory [10]. Scientists have devoted tremendous efforts to solve Bratu's problem. Babolian et al. [3] applied the reproducing kernel Hilbert space method for 
solving Bratu-type differential equations of fractional order, Laplace Adomian decomposition method and Adomian decomposition method were applied for solving Bratu's problem by Syam and Wazwaz respectively[20]. Aksoy and Pakdemirli [1] had solved Bratu-type equation of new perturbation iteration solutions. Yiming Chen et al. [7] applied the Chebyshev wavelet method for solving fractional integral and differential equations of Bratu-type, CAS wavelets was applied to study it by Mingxu Yi et al. [21].

In recent decades, many researchers have tried to study orthogonal functions for obtaining solutions of integral equations. Using orthogonal basis, a functional integral equation can be reduced into a system of algebraic equations. Researchers used several different types of orthogonal functions for finding solutions of integral equations. Comparison between these orthogonal functions, orthogonal wavelet basis are useful and powerful basis for solving functional integral equations. Over the past thirty years, scientists have studied the fractional differential equations (FDEs), because many physical phenomena in the other sciences can be modeled using the fractional derivatives. Over the last two decades, since apply of wavelets by scientists, these functions have proved their applications in study of many problems of engineering, physics and applied mathematics. Wavelets have several useful properties, such as compact support, symmetry, orthogonality and closed form. Therefore, these functions have been widely used by many researchers.

Many types of integral equations have been solved via some wavelets such as, CAS, Haar, Legendre and Chebyshev [17, 18]. Lepik applied the Haar wavelet to obtain solutions of fractional integral equations and nonlinear integro-differential equations [12,13]. The Haar wavelet is used to obtain numerical solutions of Fredholm integral equations. Chebyshev wavelet was used by Babolian to investigate differential equations[2]. Furthermore, a CAS wavelet has been used to FDEs in [18], and so on.

Many researchers used Legendre wavelets for their studies to solve differential equations [14] and references therein. Our purpose of this study, is to investigate approximate solutions of the following fractional integro-differential equations of Bratu-type by using Legendre wavelets.

$$
\begin{array}{ll}
D_{0_{+}}^{\alpha} \zeta(x)+\lambda \int_{0}^{x} k(x, t) \exp (\zeta(t)) d t=g(x), & m-1<\alpha \leq m, 0 \leq x, t \leq 1, \\
\zeta^{(j)}(0)=C_{j}, & j=0, \cdots, m-1,
\end{array}
$$

where $D_{0_{+}}^{\alpha}$ is the fractional derivative, $\zeta(x)$ is unknown function on the interval $[0,1]$, $\lambda$ and $C_{j}, j=0, \cdots, m-1$, are given constant, $m$ is a positive integer number, $k(x, t) \in$ $L^{2}([0,1] \times[0,1])$ and $g(x)$ is a known function. The main idea is to replace a FDE with a Volterra integral equation, and then provide an efficient numerical algorithm according to Legendre wavelets operational matrix. 
This paper is organized as follows. Section 2 is given some preliminaries of the fractional calculus theory. Description of the definitions of wavelets and the Legendre polynomials are given in Section 3. In Section 4, we introduce the Legendre wavelets to approximate the functions. We presented some numerical examples to illustrate validity and simplicity of the numerical approach in Section 5. The obtained numerical solutions by this method are compared with exact solutions as well. Conclusions of this paper are summarized in Section 6.

\section{PRELIMINARIES}

In this section, we illustrate fractional calculus, which are used throughout this paper [15].

Definition 1. The Riemann-Liouville fractional integration operator $I_{0_{+}}^{\alpha}$ of order $\alpha(\alpha \geq 0)$ and Caputo fractional derivative operator $D_{0_{+}}^{\alpha}$ of order $\alpha(\alpha \geq 0)$ on the usual Lebesgue space $L[0, T]$ are defined as:

- Riemann-Liouville fractional integration operator:

$$
I_{0_{+}}^{\alpha} \zeta(t)=\frac{1}{\Gamma(\alpha)} \int_{0}^{t}(t-\tau)^{(\alpha-1)} \zeta(\tau) d \tau, I_{0_{+}}^{0} \zeta(t)=\zeta(t), t>0,
$$

- Caputo fractional derivative operator:

$$
D_{0_{+}}^{\alpha} \zeta(t)=\frac{1}{\Gamma(m-\alpha)} \int_{0}^{t}(t-\tau)^{m-\alpha-1} \zeta^{(m)}(\tau) d \tau, \quad m-1<\alpha \leq m, \quad t>0 .
$$

where $m$ is a positive integer number and $\Gamma($.$) is well-known Euler's gamma function.$

Some of the basic and main properties of the Riemann-Liouville fractional integral and Caputo fractional derivative operators are given below:

(i) $I_{0_{+}}^{\alpha} I_{0_{+}}^{\mu} \zeta(t)=I_{0_{+}}^{\alpha+\mu} \zeta(t)$,

(ii) $I_{0_{+}}^{\alpha} I_{0_{+}}^{\mu} \zeta(t)=I_{0_{+}}^{\mu} I_{0_{+}}^{\alpha} \zeta(t)$,

(iii) $I_{0_{+}}^{\alpha} t^{\mu}=\frac{\Gamma(\mu+1)}{\Gamma(\alpha+\mu+1)} t^{\alpha+\mu}$,

(iv) $I_{0_{+}}^{\alpha} D_{0_{+}}^{\alpha} \zeta(t)=\zeta(t)-\sum_{q=0}^{m-1} \zeta^{(q)}\left(0^{+}\right) \frac{t^{q}}{q !}, \quad t>0$

(v) $D_{0_{+}}^{\alpha} J_{0_{+}}^{\alpha} \zeta(t)=\zeta(t)$,

(vi) $\int_{0}^{1} \tau^{\alpha-1}(1-\tau)^{\mu-1} d \tau=\frac{\Gamma(\alpha) \Gamma(\mu)}{\Gamma(\alpha+\mu)}$.

\section{LEGENDRE WAVELETS}

Wavelets constitute of a family of functions constructed by translation and dilation of a single function called mother wavelet $\psi(x)$, which is defined as follows:

$$
\psi_{a, b}(x)=\frac{1}{\sqrt{|a|}} \psi\left(\frac{x-b}{a}\right), \quad a, b \in \mathbb{R}, \quad a \neq 0 .
$$

Here $a$ and $b$ are the dilation and translation parameters.

By restricting the parameters $a$ and $b$ to discrete values as $a=a_{0}^{-j}, b=k b_{0} a_{0}^{-j}$ where 
$a_{0}>1, b_{0}>1, j, k \in \mathbb{N}$, we obtain a family of discrete wavelets as:

$$
\psi_{j, k}(x)=\left|a_{0}\right|^{\frac{j}{2}} \psi\left(a_{0}^{j} x-k b_{0}\right) .
$$

If $a_{0}=2$ and $b_{0}=1$, the above $\psi_{j, k}(x)$ may construct an orthonormal basis for certain $\psi$, that is

$$
\left\langle\psi_{j, k}, \psi_{l, m}\right\rangle=\delta_{j l} \delta_{k m}
$$

where $\delta_{j l}$ indicates the Kronecker delta.

The Legendre polynomial of order $m$ can be defined by the following recurrence formula on the interval $[-1,1]$

$$
\begin{aligned}
& p_{0}(x)=1, \\
& p_{1}(x)=x, \\
& p_{m+1}(x)=\frac{2 m+1}{m+1} x p_{m}(x)-\frac{m}{m+1} p_{m-1}(x) \quad m=1,2,3, \ldots
\end{aligned}
$$

Legendre wavelets defined on the interval $[0,1)$ as:

$$
\psi_{n, m}(x)=\left\{\begin{array}{lr}
(2 m+1)^{\frac{1}{2}} 2^{\frac{k}{2}} p_{m}\left(2^{k} x-\hat{n}\right), & \frac{\hat{n}-1}{2^{k}} \leq x<\frac{\hat{n}+1}{2^{k}}, \\
0, & \text { otherwise },
\end{array}\right.
$$

where $n=1,2,3, \ldots, 2^{k-1}, k=2,3, \ldots, \hat{n}=2 n-1$ and $m=0,1,2, \ldots, M-1 . m$ is the order of Legendre polynomials and $M$ is a positive integer.

\section{FUnCTIONS APPROXIMATION BY LEGENDRE WAVELETS}

For any function $u(x)$ with square integrable on $[0,1)$, we can express it in terms of the Legendre wavelet polynomials as follows:

$$
u(x)=\sum_{n=1}^{\infty} \sum_{m=0}^{\infty} c_{n m} \psi_{n, m}(x),
$$

where

$$
c_{n m}=\left\langle u(x), \psi_{n, m}(x)\right\rangle .
$$

If the infinite series in (4.1) is truncated, then equation (4.1) can be written as follows:

$$
u(x) \cong u_{\hat{m}}(x)=\sum_{n=1}^{2^{k-1}} \sum_{m=0}^{M-1} c_{n m} \psi_{n, m}(x)=C^{T} \Psi(x),
$$

where $C$ and $\Psi(x)$ are two $\hat{m} \times 1\left(\hat{m}=2^{k-1} M\right)$ matrices given by

$$
\begin{gathered}
C=\left[c_{10}, c_{11}, \ldots, c_{1 M-1}, c_{20}, c_{21}, \ldots, c_{2 M-1}, \ldots, c_{2^{k-1} 0}, \ldots, c_{2^{k-1} M-1}\right]^{T}, \\
\Psi(x)=\left[\psi_{10}, \psi_{11}, \ldots, \psi_{1 M-1}, \psi_{20}, \ldots, \psi_{2 M-1}, \ldots, \psi_{2^{k-1} 0}, \ldots, \psi_{2^{k-1} M-1}\right]^{T} .
\end{gathered}
$$


For simplicity the equation (4.2) can be rewritten as follows:

$$
u(x) \cong u_{\hat{m}}(x)=\sum_{i=1}^{\hat{m}} c_{i} \psi_{i}(x)=C^{T} \Psi(x)
$$

where $c_{i}=c_{n m}, \psi_{i}(x)=\psi_{n m}, i=M(n-1)+m+1$. Here, we rewrite the matrices $\Psi$ and $C$ as follows:

$$
C=\left[c_{1}, c_{2}, \ldots, c_{\hat{m}}\right]^{T}, \quad \Psi(x)=\left[\psi_{1}(x), \ldots, \psi_{\hat{m}}(x)\right]^{T} .
$$

Similarly, for expanding of two variables functions such as $k(x, y) \in L^{2}([0,1] \times[0,1])$ in terms of Legendre wavelet functions we can write:

$$
k(x, y) \cong \sum_{i=1}^{\hat{m}} \sum_{j=1}^{\hat{m}} k_{i j} \psi_{i}(x) \psi_{j}(y)=\Psi(x)^{T} K \Psi(y)
$$

where $K_{\hat{m} \times \hat{m}}$ is given as follows:

$$
K=\left[k_{i j}\right]_{\hat{m} \times \hat{m}}, \quad k_{i j}=\left\langle\psi_{i}(x),\left\langle k(x, y), \psi_{j}(y)\right\rangle\right\rangle, \quad i, j=1,2, \ldots, \hat{m} .
$$

Theorem 1. For solving Fredholm-Volterra integral equation, the operational matrix $P$ of integration is given as follows

$$
P_{\hat{m} \times \hat{m}}=\left[\begin{array}{ccccc}
L & F & F & \ldots & F \\
0 & L & F & \ldots & F \\
0 & 0 & L & \ldots & F \\
\vdots & \vdots & \vdots & \ddots & \vdots \\
0 & 0 & 0 & \cdots & L
\end{array}\right]
$$

where $F$ and $L$ are square matrices of order $M$ given by

$$
F_{M \times M}=\frac{1}{2^{k}}\left[\begin{array}{cccc}
2 & 0 & \ldots & 0 \\
0 & 0 & \ldots & 0 \\
\vdots & \vdots & \vdots & \vdots \\
0 & 0 & 0 & 0
\end{array}\right]
$$




$$
\begin{aligned}
& L_{M \times M}= \\
& \frac{1}{2^{k}}\left[\begin{array}{ccccccc}
1 & \frac{1}{\sqrt{3}} & 0 & 0 & \ldots & 0 & 0 \\
-\frac{\sqrt{3}}{3} & 0 & \frac{\sqrt{3}}{3 \sqrt{5}} & 0 & \ldots & 0 & 0 \\
0 & -\frac{\sqrt{5}}{5 \sqrt{3}} & 0 & \frac{\sqrt{5}}{5 \sqrt{7}} & \cdots & 0 & 0 \\
0 & 0 & -\frac{\sqrt{7}}{7 \sqrt{5}} & 0 & \ldots & 0 & 0 \\
\vdots & \vdots & \vdots & \vdots & \ddots & \vdots & \vdots \\
0 & 0 & 0 & 0 & \ldots & 0 & \sqrt{2 M-3} \\
0 & 0 & 0 & 0 & \ldots & -\frac{\sqrt{2 M-1}}{(2 M-1) \sqrt{2 M-3}} & 0
\end{array}\right]
\end{aligned}
$$

Proof. First we get the operational matrix of integration for $k=2$ and $M=3$. Then we give the general matrix $P$. The basis functions $\psi_{n m}(x), n=1,2, m=0,1,2$ and consequently the matrix

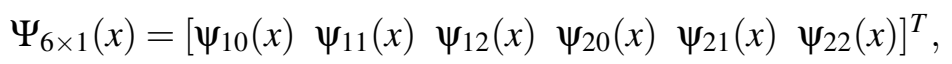

for $k=2$ and $M=3$ are computed as

$$
\left.\begin{array}{l}
\psi_{10}(x)=\sqrt{2} \\
\psi_{11}(x)=\sqrt{6}(4 x-1), \\
\psi_{12}(x)=\sqrt{10}\left[\frac{3}{2}(4 x-1)^{2}-\frac{1}{2}\right],
\end{array}\right\} \quad 0 \leq x<\frac{1}{2},
$$

and

$$
\left.\begin{array}{l}
\psi_{20}(x)=\sqrt{2}, \\
\psi_{21}(x)=\sqrt{6}(4 x-3), \\
\psi_{22}(x)=\sqrt{10}\left[\frac{3}{2}(4 x-3)^{2}-\frac{1}{2}\right],
\end{array}\right\} \quad \frac{1}{2} \leq x<1,
$$

By integrating (4.3) and (4.4), we obtain the following relations:

$$
\begin{array}{ll}
\int_{0}^{x} \psi_{10}(t) d t= \begin{cases}\sqrt{2} x, & 0 \leq x<\frac{1}{2}, \\
\frac{1}{\sqrt{2}}, & \frac{1}{2} \leq x<1,\end{cases} \\
=\frac{1}{4} \Psi_{10}(x)+\frac{\sqrt{2}}{4 \sqrt{6}} \psi_{11}(x)+\frac{1}{2} \Psi_{20}(x)=\left[\frac{1}{4}, \frac{\sqrt{2}}{4 \sqrt{6}}, 0, \frac{1}{2}, 0,0\right] \Psi_{6 \times 1}(x) .
\end{array}
$$




$$
\begin{aligned}
& \int_{0}^{x} \psi_{11}(t) d t= \begin{cases}2 \sqrt{6} x^{2}-\sqrt{6} x, & 0 \leq x<\frac{1}{2}, \\
0, & \frac{1}{2} \leq x<1,\end{cases} \\
& =-\frac{\sqrt{3}}{12} \psi_{10}(x)+\frac{\sqrt{3}}{12 \sqrt{5}} \psi_{12}(x)=\left[-\frac{\sqrt{3}}{12}, 0, \frac{\sqrt{3}}{12 \sqrt{5}}, 0,0,0\right] \Psi_{6 \times 1}(x) .
\end{aligned}
$$

Similarly, we have

$$
\begin{aligned}
& \int_{0}^{x} \Psi_{12}(t) d t=-\frac{\sqrt{5}}{20 \sqrt{3}} \Psi_{11}(x)=\left[0,-\frac{\sqrt{5}}{20 \sqrt{3}}, 0,0,0,0\right] \Psi_{6 \times 1}(x), \\
& \int_{0}^{x} \Psi_{20}(t) d t=\frac{1}{4} \Psi_{20}(x)+\frac{\sqrt{2}}{4 \sqrt{6}} \Psi_{21}(x)=\left[0,0,0, \frac{1}{4}, \frac{\sqrt{2}}{4 \sqrt{6}}, 0\right] \Psi_{6 \times 1}(x), \\
& \int_{0}^{x} \Psi_{21}(t) d t=-\frac{\sqrt{3}}{12} \Psi_{20}(x)+\frac{\sqrt{3}}{12 \sqrt{5}} \Psi_{22}(x)=\left[0,0,0,-\frac{\sqrt{3}}{12}, 0, \frac{\sqrt{3}}{12 \sqrt{5}}\right] \Psi_{6 \times 1}(x), \\
& \int_{0}^{x} \Psi_{22}(t) d t=-\frac{\sqrt{5}}{20 \sqrt{3}} \psi_{21}(x)=\left[0,0,0,0,-\frac{\sqrt{5}}{20 \sqrt{3}}, 0\right] \Psi_{6 \times 1}(x),
\end{aligned}
$$

Therefore, we have

$$
\int_{0}^{x} \Psi_{6 \times 1}(t) d t=P_{6 \times 6} \Psi_{6 \times 1}(x),
$$

where $P_{6 \times 6}$ is a square matrix of order 6 which it can be given as the following form:

$$
P_{6 \times 6}=\left[\begin{array}{ll}
L_{3 \times 3} & F_{3 \times 3} \\
0_{3 \times 3} & L_{3 \times 3}
\end{array}\right]
$$

where

$$
L_{3 \times 3}=\frac{1}{2^{2}}\left[\begin{array}{ccc}
1 & \frac{\sqrt{2}}{\sqrt{6}} & 0 \\
-\frac{\sqrt{3}}{3} & 0 & \frac{\sqrt{3}}{3 \sqrt{5}} \\
0 & -\frac{\sqrt{5}}{5 \sqrt{3}} & 0
\end{array}\right] \quad \text { and } \quad F_{3 \times 3}=\frac{1}{2^{2}}\left[\begin{array}{ccc}
2 & 0 & 0 \\
0 & 0 & 0 \\
0 & 0 & 0
\end{array}\right] \text {. }
$$

If the theorem be true for M-1 and $\mathrm{K}-1$, then it is simple to show for general case $\mathrm{M}$ and $\mathrm{k}$. we have

$$
\int_{0}^{x} \Psi_{\hat{m} \times 1}(t) d t=P_{\hat{m} \times \hat{m}} \Psi_{\hat{m} \times 1}(x), \quad\left(\hat{m}=2^{k-1} M\right),
$$


Here, $P_{\hat{m} \times \hat{m}}$ can be written as follow

$$
P_{\hat{m} \times \hat{m}}=\left[\begin{array}{ccccc}
L & F & F & \ldots & F \\
0 & L & F & \ldots & F \\
0 & 0 & L & \ldots & F \\
\vdots & \vdots & \vdots & \ddots & \vdots \\
0 & 0 & 0 & \cdots & L
\end{array}\right]
$$

where $F$ and $L$ are square matrices of order $M$ given by

$$
F_{M \times M}=\frac{1}{2^{k}}\left[\begin{array}{cccc}
2 & 0 & \ldots & 0 \\
0 & 0 & \ldots & 0 \\
\vdots & \vdots & \vdots & \vdots \\
0 & 0 & 0 & 0
\end{array}\right]
$$

$$
\begin{aligned}
& L_{M \times M}= \\
& \frac{1}{2^{k}}\left[\begin{array}{ccccccc}
1 & \frac{1}{\sqrt{3}} & 0 & 0 & \ldots & 0 & 0 \\
-\frac{\sqrt{3}}{3} & 0 & \frac{\sqrt{3}}{3 \sqrt{5}} & 0 & \ldots & 0 & 0 \\
0 & -\frac{\sqrt{5}}{5 \sqrt{3}} & 0 & \frac{\sqrt{5}}{5 \sqrt{7}} & \ldots & 0 & 0 \\
0 & 0 & -\frac{\sqrt{7}}{7 \sqrt{5}} & 0 & \ldots & 0 & 0 \\
\vdots & \vdots & \vdots & \vdots & \ddots & \vdots & \vdots \\
0 & 0 & 0 & 0 & \ldots & 0 & \frac{\sqrt{2 M-3}}{(2 M-3) \sqrt{2 M-1}} \\
0 & 0 & 0 & 0 & \ldots & -\frac{\sqrt{2 M-1}}{(2 M-1) \sqrt{2 M-3}} & 0
\end{array}\right]
\end{aligned}
$$

Now, we will apply our method to problem I and II.

\section{PROBLEM I}

We demonstrate this method to the following fractional integro-differential equation of Bratu type:

$$
\begin{array}{cc}
D_{0_{+}}^{\alpha} \zeta(x)+\lambda \int_{0}^{x}(x-t)^{p} \exp (\zeta(t)) d t=g(x), & m-1<\alpha \leq m, 0 \leq x, t \leq 1, \\
\zeta^{(j)}(0)=C_{j}, & j=0, \cdots, m-1 .
\end{array}
$$

Using Riemann-Liouville fractional integration from Eq. (4.5), we have

$$
\zeta(x)+\frac{1}{\Gamma(\alpha)} \int_{0}^{x}(x-t)^{\alpha-1}\left(\lambda \int_{0}^{t}(t-\tau)^{p} \exp (\zeta(\tau)) d \tau\right) d t
$$




$$
=\frac{1}{\Gamma(\alpha)} \int_{0}^{x}(x-t)^{\alpha-1} g(t) d t .
$$

We first rewrite Eq. (4) as

$$
\zeta(x)=G(x)-\frac{\lambda}{\Gamma(\alpha)} \int_{0}^{x} \int_{0}^{t}(x-t)^{\alpha-1}(t-\tau)^{p} \exp (\zeta(\tau)) d \tau d t
$$

where $G(x)=\frac{1}{\Gamma(\alpha)} \int_{0}^{x}(x-t)^{\alpha-1} g(t) d t$. However, by changing the order of integration in Eq. (4), we have

$$
\zeta(x)=G(x)-\frac{\lambda}{\Gamma(\alpha)} \int_{0}^{x}\left(\int_{\tau}^{x}(x-t)^{\alpha-1}(t-\tau)^{p} d t\right) \exp (\zeta(\tau)) d \tau,
$$

Now, using the change of variables $s=\frac{x-t}{x-\tau}$, and according to $\int_{0}^{1} s^{\alpha-1}(1-s)^{p} d s=$ $\frac{\Gamma(\alpha) \Gamma(p+1)}{\Gamma(\alpha+p+1)}$, Eq. (4.7) is converted to following Volterra integral equation

$$
\zeta(x)=G(x)-\frac{\lambda \Gamma(p+1)}{\Gamma(\alpha+p+1)} \int_{0}^{x}(x-\tau)^{\alpha+p} \exp (\zeta(\tau)) d \tau .
$$

We rewrite Eq. (4.8) as

$$
\zeta(x)=F(x, \zeta(x)), \quad 0 \leq x \leq 1,
$$

where $F(x, \zeta(x))=G(x)-\frac{\lambda \Gamma(p+1)}{\Gamma(\alpha+p+1)} \int_{0}^{x}(x-\tau)^{\alpha+p} \exp (\zeta(\tau)) d \tau$.

\section{PROBLEM II}

Consider the following fractional integro-differential equation of Bratu type:

$$
\begin{array}{ll}
D_{0_{+}}^{\alpha} \zeta(x)+\lambda \int_{0}^{x}(x-a)^{p}(t-b)^{q} \exp (\zeta(t)) d t=g(x), & m-1<\alpha \leq m, \\
& 0 \leq x, t \leq 1, \\
\zeta^{(j)}(0)=C_{j}, & j=0, \cdots, m-1 .
\end{array}
$$

Using Riemann-Liouville fractional integration from Eq. (4.10), we have

$$
\begin{aligned}
& \zeta(x)+\frac{1}{\Gamma(\alpha)} \int_{0}^{x}(x-t)^{\alpha-1}\left(\lambda \int_{0}^{t}(t-a)^{p}(\tau-b)^{q} \exp (\zeta(\tau)) d \tau\right) d t \\
& =\frac{1}{\Gamma(\alpha)} \int_{0}^{x}(x-t)^{\alpha-1} g(t) d t
\end{aligned}
$$

We first rewrite Eq. (4.11) as

$$
\zeta(x)=G(x)-\frac{\lambda}{\Gamma(\alpha)} \int_{0}^{x} \int_{0}^{t}(x-t)^{\alpha-1}(t-a)^{p}(\tau-b)^{q} \exp (\zeta(\tau)) d \tau d t,
$$


where $G(x)=\frac{1}{\Gamma(\alpha)} \int_{0}^{x}(x-t)^{\alpha-1} g(t) d t$. However, by changing the order of integrations in Eq. (4.11), we have

$$
\zeta(x)=G(x)-\frac{\lambda}{\Gamma(\alpha)} \int_{0}^{x}(\tau-b)^{q}\left(\int_{\tau}^{x}(x-t)^{\alpha-1}(t-a)^{p} d t\right) \exp (\zeta(\tau)) d \tau,
$$

then, using the change of variables $s=\frac{x-t}{x-\tau}$, and according to $\int_{0}^{1} s^{\alpha-1}(1-s)^{p-k} d s=$ $\frac{\Gamma(\alpha) \Gamma(p-k+1)}{\Gamma(\alpha+p-k+1)}$, Eq. (4.12) is converted to following Volterra integral equation

$$
\zeta(x)=G(x)-\sum_{k=0}^{p} \frac{\lambda p !}{k ! \Gamma(\alpha+p-k+1)} \int_{0}^{x}(x-\tau)^{\alpha+p-k}(\tau-a)^{k}(\tau-b)^{q} \exp (\zeta(\tau)) d \tau .
$$

We rewrite Eq. (4.13) as

$$
\zeta(x)=F(x, \zeta(x)), \quad 0 \leq x \leq 1,
$$

where

$$
F(x, \zeta(x))=G(x)-\sum_{k=0}^{p} \frac{\lambda p !}{k ! \Gamma(\alpha+p-k+1)} \int_{0}^{x}(x-\tau)^{\alpha+p-k}(\tau-a)^{k}(\tau-b)^{q} \exp (\zeta(\tau)) d \tau .
$$

Theorem 2. The fractional integro-differential equations of Bratu type Eq. (1.1) as well as Eqs. (4.9) and (4.14) have a unique solutions([5]).

\section{NUMERICAL EXAMPLES}

In this section, in order to test the validity of our method, three examples are solved and the numerical results are compared with their exact solution.

Example 1. Assume the following fractional integro-differential equations of Bratutype:

$$
\begin{cases}D_{0_{+}}^{\alpha} \zeta(x)+3 \int_{0}^{x}(x-t) \exp (\zeta(t)) d t=\frac{1}{x+1}+\frac{1}{2} x^{3}+\frac{3}{2} x^{2}, & 0<x, t<1, \\ \zeta(0)=0, & 0<\alpha \leq 1 .\end{cases}
$$

The exact solution for $\alpha=1$ is $\zeta(x)=\ln (x+1)$. The numerical results for the exact solution of this example for $\alpha=1$ and approximate solutions for $\alpha=0.5,0.6,0.7,0.8$, $0.9,1.0, k=2, M=7$ are shown in Fig. 1 .

Example 2. We assume the following fractional integro-differential equations of Bratu-type:

$$
\begin{cases}D_{0_{+}}^{\alpha} \zeta(x)+\int_{0}^{x} x\left(t-\frac{1}{2}\right) \exp (\zeta(t)) d t= & \frac{\Gamma(3)}{\Gamma(2.5)} x^{1.5}-\frac{\Gamma(2)}{\Gamma(1.5)} x^{0.5} \\ +\frac{x}{2}\left(\exp \left(x^{2}-x\right)-1\right), & 0<x, t<1, \\ \zeta(0)=0, & 0<\alpha \leq 1 .\end{cases}
$$




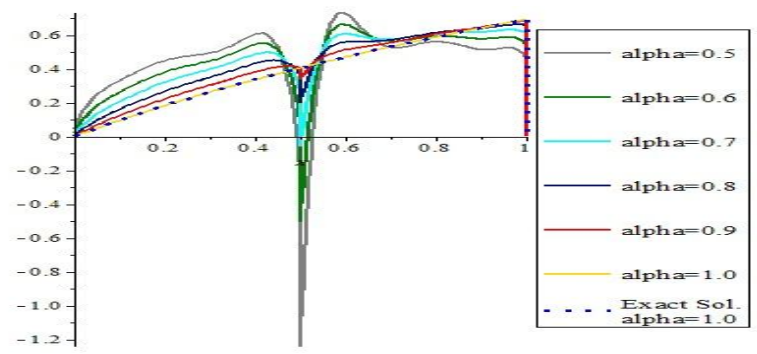

FIGURE 1. Comparison between the exact solution for $\alpha=1$ and approximate solutions for $\alpha=0.2,0.3,0.40 .5,0.6,0.7,0.8, k=2, M=7$ for Example 1.

The exact solution for $\alpha=0.5$ is $\zeta(x)=x^{2}-x$. The numerical results for the exact solution of this example for $\alpha=0.5$ and approximate solutions for $\alpha=0.5,0.6,0.7$, $0.8,0.9,1.0, k=2, M=7$ are shown in Fig. 2.

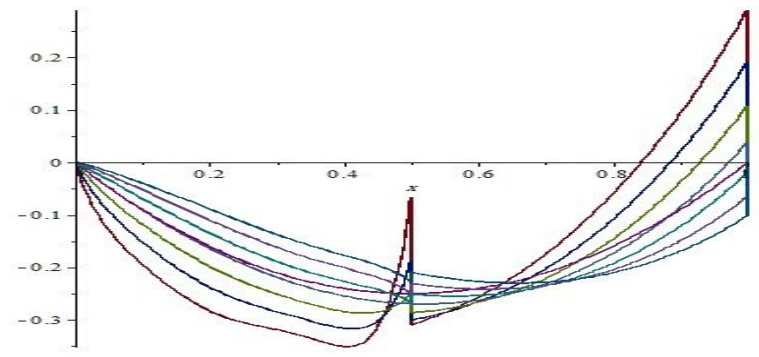

FIGURE 2. Comparison between the exact solution for $\alpha=0.5$ and approximate solutions for $\alpha=0.2,0.3,0.40 .5,0.6,0.7,0.8, k=2, M=7$ for Example 2.

Example 3. We consider the following fractional integro-differential equations of Bratu-type:

$$
\left\{\begin{array}{l}
D_{0_{+}}^{0.75} \zeta(x)+\frac{1}{4} \int_{0}^{x}(x-t) \exp (\zeta(t)) d t=\frac{8 \sqrt[4]{x}}{\Gamma(0.25)}+\frac{\exp (2 x)-2 x-1}{16}, 0<x, t<1, \\
\zeta(0)=0 .
\end{array}\right.
$$


The exact solution for $\alpha=0.75$ is $\zeta(x)=2 x$. The numerical results for the exact solution of this example for $\alpha=0.75$ and approximate solutions for $\alpha=0.5,0.6,0.7,0.8$, $0.9,1.0, k=2, M=7$ are shown in Fig. 3 .

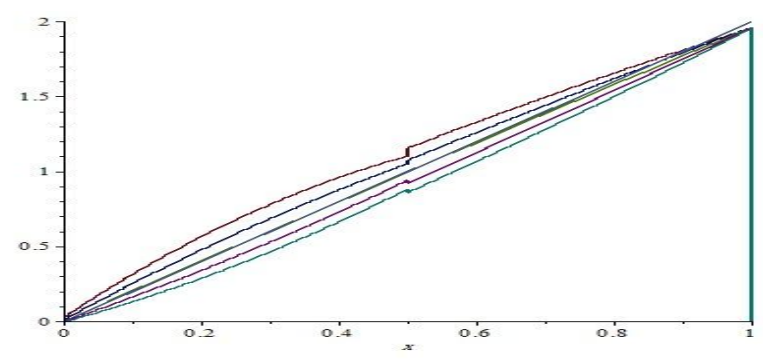

FIGURE 3. Comparison between the exact solution for $\alpha=0.5$ and approximate solutions for $\alpha=0.2,0.3,0.40 .5,0.6,0.7,0.8, k=2, M=7$ for Example 3

\section{CONCLUding REMARKS}

The aim of this paper is to improve an efficient and precise method for solving non-linear fractional integro-differential equations of Bratu-type. In this paper, in addition to use the properties of the Legendre wavelets, we use the Gauss quadrature rules. Then, we converted the problem into a system of nonlinear algebraic equations. Finally, we applied the Newton iteration method for solving the resulted system. Examples are provided to explain and interpret the simplicity and applicability of this technique.

\section{ACKNOWLEDGEMENT}

M.F. is partially supported by the Grants Slovak Research and Development Agency under the contract No. APW-18-0308 and by the Slovak Grant Agency VEGA No. 1/0358/20 and No. 2/0127/20.

\section{REFERENCES}

[1] Y. Aksoy and M. Pakdemirli, "New perturbation-iteration solutions for bratu-type equations," Computers \& Mathematics with Applications, vol. 59, no. 8, pp. 2802-2808, 2010.

[2] E. Babolian and F. Fattahzadeh, "Numerical solution of differential equations by using chebyshev wavelet operational matrix of integration," Applied Mathematics and computation, vol. 188, no. 1, pp. 417-426, 2007. 
[3] E. Babolian, S. Javadi, and E. Moradi, "RKM for solving bratu-type differential equations of fractional order," Mathematical Methods in the Applied Sciences, vol. 39, no. 6, pp. 1548-1557, 2016.

[4] T. Blaszczyk and M. Ciesielski, "Numerical solution of fractional sturm-liouville equation in integral form," Fractional Calculus and Applied Analysis, vol. 17, no. 2, pp. 307-320, Jun 2014, doi: 10.2478/s13540-014-0170-8. [Online]. Available: https://doi.org/10.2478/ s13540-014-0170-8

[5] C.-M. Chen, F. Liu, V. Anh, and I. Turner, "Numerical schemes with high spatial accuracy for a variable-order anomalous subdiffusion equation," SIAM Journal on Scientific Computing, vol. 32, no. 4, pp. 1740-1760, 2010.

[6] W. Chen, "A speculative study of 2/3-order fractional laplacian modeling of turbulence: Some thoughts and conjectures," Chaos, vol. 16, no. 2, p. 023126, Jun. 2006, doi: 10.1063/1.2208452.

[7] Y. M. Chen, L. Sun, L. Liu, and J. Xie, "The chebyshev wavelet method for solving fractional integral and differential equations of bratu-type," J. Comput. Inf. Syst, vol. 9, no. 14, pp. 5601$5609,2013$.

[8] M. Dehghan, J. Manafian, and A. Saadatmandi, "Solving nonlinear fractional partial differential equations using the homotopy analysis method," Numer. Methods Partial Differ. Eqn., vol. 26, no. 2, pp. 448-479, 2009.

[9] M. R. Eslahchi, M. Dehghan, and M. Parvizi, "Application of the collocation method for solving nonlinear fractional integro-differential equations," Journal of Computational and Applied Mathematics, vol. 257, pp. 105-128, 2014.

[10] I. H. A. H. Hassan and V. S. Ertürk, "Applying differential transformation method to," Int. J. Contemp. Math. Sciences, vol. 2, no. 30, pp. 1493-1504, 2007.

[11] M. Lakestani, M. Jokar, and M. Dehghan, "Numerical solution of nth-order integro-differential equations using trigonometric wavelets," Mathematical Methods in the Applied Sciences, vol. 34, no. 11, pp. 1317-1329, 2011.

[12] Ü. Lepik, "Haar wavelet method for nonlinear integro-differential equations," Applied mathematics and Computation, vol. 176, no. 1, pp. 324-333, 2006.

[13] Ü. Lepik, "Solving fractional integral equations by the haar wavelet method," Applied Mathematics and Computation, vol. 214, no. 2, pp. 468-478, 2009.

[14] Z. Meng, M. Yi, J. Huang, and L. Song, "Numerical solutions of nonlinear fractional differential equations by alternative legendre polynomials," Applied Mathematics and Computation, vol. 336, pp. 454-464, 2018.

[15] K. S. Miller and B. Ross, An introduction to the fractional calculus and fractional differential equations. New York: John Wiley \& Sons, Inc., 1993.

[16] Z. Odibat and S. Momani, "A generalized differential transform method for linear partial differential equations of fractional order," Applied Mathematics Letters, vol. 21, no. 2, pp. 194-199, 2008.

[17] U. Saeed, "Cas picard method for fractional nonlinear differential equation," Applied Mathematics and Computation, vol. 307, pp. 102-112, 2017.

[18] H. Saeedi and M. M. Moghadam, "Numerical solution of nonlinear volterra integro-differential equations of arbitrary order by cas wavelets," Communications in Nonlinear Science and Numerical Simulation, vol. 16, no. 3, pp. 1216-1226, 2011.

[19] H. Sun, W. Chen, H. Sheng, and Y. Chen, "On mean square displacement behaviors of anomalous diffusions with variable and random orders," Physics Letters A, vol. 374, no. 7, pp. 906-910, 2010.

[20] A.-M. Wazwaz, "Adomian decomposition method for a reliable treatment of the bratu-type equations," Applied Mathematics and Computation, vol. 166, no. 3, pp. 652-663, 2005.

[21] M. Yi, K. Sun, J. Huang, and L. Wang, "Numerical solutions of fractional integrodifferential equations of bratu type by using cas wavelets," Journal of Applied Mathematics, vol. 2013, 2013. 
[22] P. Zhuang, F. Liu, V. Anh, and I. Turner, "Numerical methods for the variable-order fractional advection-diffusion equation with a nonlinear source term," SIAM Journal on Numerical Analysis, vol. 47, no. 3, pp. 1760-1781, 2009.

\section{Authors' addresses}

\section{Felahat}

Department of Mathematics, Graduate University of Advanced Technology, Kerman, Iran

E-mail address: m. felahat@yahoo.com

\section{N. Kadkhoda}

Department of Mathematics, Faculty of Basic Sciences, Bozorgmehr University Of Qaenat, Qaenat, Iran

E-mail address: kadkhoda@buqaen.ac.ir

\section{Fečkan}

(Corresponding author) Department of Mathematical Analysis and Numerical Mathematics, Faculty of Mathematics, Physics and Informatics, Comenius University in Bratislava, Mlynska dolina, 84248 Bratislava, Slovakia

Mathematical Institute of Slovak Academy of Sciences, Štefánikova 49, 814 73, Bratislava, Slovakia

E-mail address: michal. feckanegmail.com 